\title{
Profiling and targeting HER2-positive breast cancer using trastuzumab emtansine
}

This article was published in the following Dove Press journal:

Pharmacogenomics and Personalized Medicine

15 October 2014

Number of times this article has been viewed

\section{Saeed Sadeghi \\ Olga Olevsky \\ Sara A Hurvitz}

Division of Hematology \& Oncology, David Geffen School of Medicine, University of California, Los Angeles, CA, USA
Correspondence: Sara A Hurvitz University of California, Los Angeles, 10945 Le Conte Ave, PVUB Suite 3360, Los Angeles, CA 90095, USA

$\mathrm{Tel}+|\mathrm{I}| 0829547 \mid$

$\mathrm{Fax}+13108296192$

Email shurvitz@mednet.ucla.edu
Purpose: This article reviews the mechanism of action of trastuzumab emtansine (T-DM1), existing clinical data relating to its use for human growth factor receptor 2 (HER2)-positive breast cancer, potential pathways of resistance, and ongoing studies evaluating this novel agent.

Background: The development of HER2-targeted therapies has dramatically improved clinical outcomes for patients with any stage of HER2-positive breast cancer. Although the positive effect of these treatments cannot be overstated, treatment resistance develops in the vast majority of those diagnosed with stage IV HER2-positive breast cancer. Moreover, HER2-directed therapies are most effective when combined with cytotoxic chemotherapy. The need for chemotherapy leads to significant adverse effects and a clear decrease in quality of life for those dealing with a chronic incurable disease. T-DM1 is a recently developed, novel antibody-drug conjugate in which highly potent maytanisinoid chemotherapy is stably linked to the HER2-targeted monoclonal antibody, trastuzumab.

Results: Preclinical and phase 1-3 clinical data support the significant antitumor activity of T-DM1. Importantly, several randomized studies also now demonstrate its clear superiority in terms of tolerability compared with standard chemotherapy-containing regimens. Its role in the treatment of trastuzumab-resistant metastatic breast cancer has now been established on the basis of the results of two phase 3 randomized studies, EMILIA (An Open-label Study of Trastuzumab Emtansine (T-DM1) vs Capecitabine + Lapatinib in Patients With HER2-positive Locally Advanced or Metastatic Breast Cancer) and TH3RESA (A Study of Trastuzumab Emtansine in Comparison With Treatment of Physician's Choice in Patients With HER2-positive Breast Cancer Who Have Received at Least Two Prior Regimens of HER2-directed Therapy). The most common toxicities seen with T-DM1 are thrombocytopenia and an elevation in liver transaminases. Significant cardiac toxicity has not been demonstrated. Both in vitro cell linebased studies as well as exploratory analyses of archived tumor samples from the clinical trials are seeking to understand potential mechanisms of resistance to T-DM1. Ongoing studies are also evaluating the use of T-DM1 in the first-line metastatic, neoadjuvant, and adjuvant settings, as well as in combination with other targeted therapies.

Conclusion: T-DM1 represents the first successfully developed antibody drug conjugate for the treatment of HER2-positive advanced breast cancer.

Keywords: HER2, T-DM1, trastuzumab emtansine, ado-trastuzumab emtansine, metastatic breast cancer, Kadcyla

\section{Introduction}

Nearly three decades have passed since Slamon and colleagues demonstrated that amplification of the gene for human epidermal human growth factor receptor 2 (HER2) is associated with a more aggressive disease course and worse clinical 
outcomes in patients with breast cancer. ${ }^{1}$ Trastuzumab (Herceptin; Genentech, South San Francisco, CA, USA), the first monoclonal antibody developed to target HER2, was shown to significantly improve the time to progression and overall survival (OS) of patients with metastatic HER2+ breast cancer. ${ }^{2}$ Significant disease-free survival and OS benefits were subsequently demonstrated in patients with localized HER2-positive (HER2+) disease with the addition of trastuzumab to standard adjuvant chemotherapy. ${ }^{3-5}$ In fact, trastuzumab has literally altered the natural history of HER2 overexpressing disease in both the metastatic and early disease settings, such that the survival for patients with trastuzumab-treated HER2+ breast cancer now mirrors, and may exceed, that of HER2-negative breast cancer. ${ }^{6,7}$ Despite the substantial activity of trastuzumab, resistance to therapy arises in the majority of patients with advanced disease. Lapatinib (Tykerb; GlaxoSmithKline, Brentford, United Kingdom), an orally bioavailable reversible inhibitor of the HER1 and HER2 tyrosine kinase domains, was subsequently shown to improve the time to progression for patients with HER2+ trastuzumab-pretreated metastatic breast cancer (MBC), ${ }^{8}$ leading to the US Food and Drug Administration (FDA) approving lapatinib plus capecitabine in this disease setting. Although this therapy provides patients with trastuzumab-resistant disease an additional treatment option, only $29 \%$ of patients had clinical benefit (complete response, partial response, or stable disease lasting at least 6 months), and half of patients had disease progression at 6.2 months. $^{9}$ A variety of resistance mechanisms has been proposed including activation of alternative signaling pathways, receptor masking, truncated HER2, or expression of the ligands of other members of the epidermal growth factor receptor family. ${ }^{10}$ Therefore, there is a continued need to develop novel agents to target HER2-amplified disease. In 2013, the FDA approved the first successful HER2-targeted antibody-drug conjugate (ADC), trastuzumab emtansine (T-DM1; Kadcyla; Genentech), for the treatment of HER2+ trastuzumab-pretreated advanced breast cancer. This article reviews the mechanism of action of T-DM1, as well as existing clinical data, ongoing studies, and potential pathways of resistance to this novel agent.

\section{Mechanism of action of T-DMI}

T-DM1 is a novel ADC composed of trastuzumab connected via a stable thioether linker to a highly potent antimicrotubule derivative of maytansine 1 (DM1; N-methyl-N-[3mercapto-1-oxopropyl]-1-alanine ester of maytansinol). DM1 is an inhibitor of microtubule dimerization, similar to vinka alkaloids, chosen as the cytotoxic component of T-DM1, given its high potency. In vitro studies have shown that in cancer cell lines, DM1 is 25-270 times more potent than paclitaxel. ${ }^{11}$ On average, 3.5 moieties of DM1 are linked via a nonreducible thioether bond (4-[N-maleimidomethyl] cyclohexane-1-carboxylate) to each trastuzumab antibody. ${ }^{12}$ T-DM1 is not the first ADC developed clinically; however, it is unique in that it is the first to use the 4-(N-maleimidomethyl) cyclohexane-1-carboxylate linker. Prior ADCs were designed using disulfide bond linkers, but experimental work showed that the oxidizing potential of lysosomes limited the cleavage of the disulfide bond within the cell. ${ }^{13}$ The 4-(N-maleimidomethyl) cyclohexane-1-carboxylate linker is advantageous, as it undergoes proteolytic digestion once internalized, and furthermore, given its greater stability, it is safer, as it limits the release of DM1 into the circulation. ${ }^{14}$ On binding the extracellular domain of HER2, T-DM1 enters the cell by the process of receptor-mediated endocytosis, and DM1 dissociates from the linker by proteolysis, presumably in the lysosome. ${ }^{15}$ In the cell cytoplasm, DM1 disrupts microtubule dynamics leading to cell cycle arrest and induction of apoptosis. ${ }^{14}$ In addition to being a cytotoxic agent, T-DM1 maintains the activity of trastuzumab by inhibiting HER2-directed signal transduction as well as activating antibody-dependent cell-mediated cytotoxicity (Figure 1). ${ }^{11}$ Preclinical work by Lewis Phillips and colleagues in a mouse xenograft model demonstrated that although T-DM1 had minimal cytotoxic effects in breast cancer cell lines lacking HER2 overexpression, it was capable of eliciting cell death in HER2 overexpressing cell lines, including those resistant to trastuzumab. ${ }^{12}$ Pharmacokinetic evaluation across multiple studies shows that the half-life of T-DM1 is approximately 4 days, and the clearance of the drug is predominantly via the biliary and gastrointestinal systems. ${ }^{16}$

The HER2-targeted nature of T-DM1 should theoretically limit the delivery of the cytotoxic component to HER2+ cells. Despite this, thrombocytopenia has been observed in all clinical trials of T-DM1 to date. Mahapatra and colleagues studied the effects of T-DM1 on platelet formation from hematopoietic stem cells as well as platelet function in vitro and demonstrated that T-DM1 is internalized in a HER2-independent fashion into megakaryocytes and that the intracellular release of DM1 leads to a reduction in the stem cell population. ${ }^{17}$ More recently, Thon and coworkers evaluated the rate and extent of megakaryocyte differentiation and proplatelet production in vitro, using a novel high-content, live cell imaging assay. Their work confirmed that T-DM1 is endocytosed into megakaryocytes and that DM1 disrupts the 


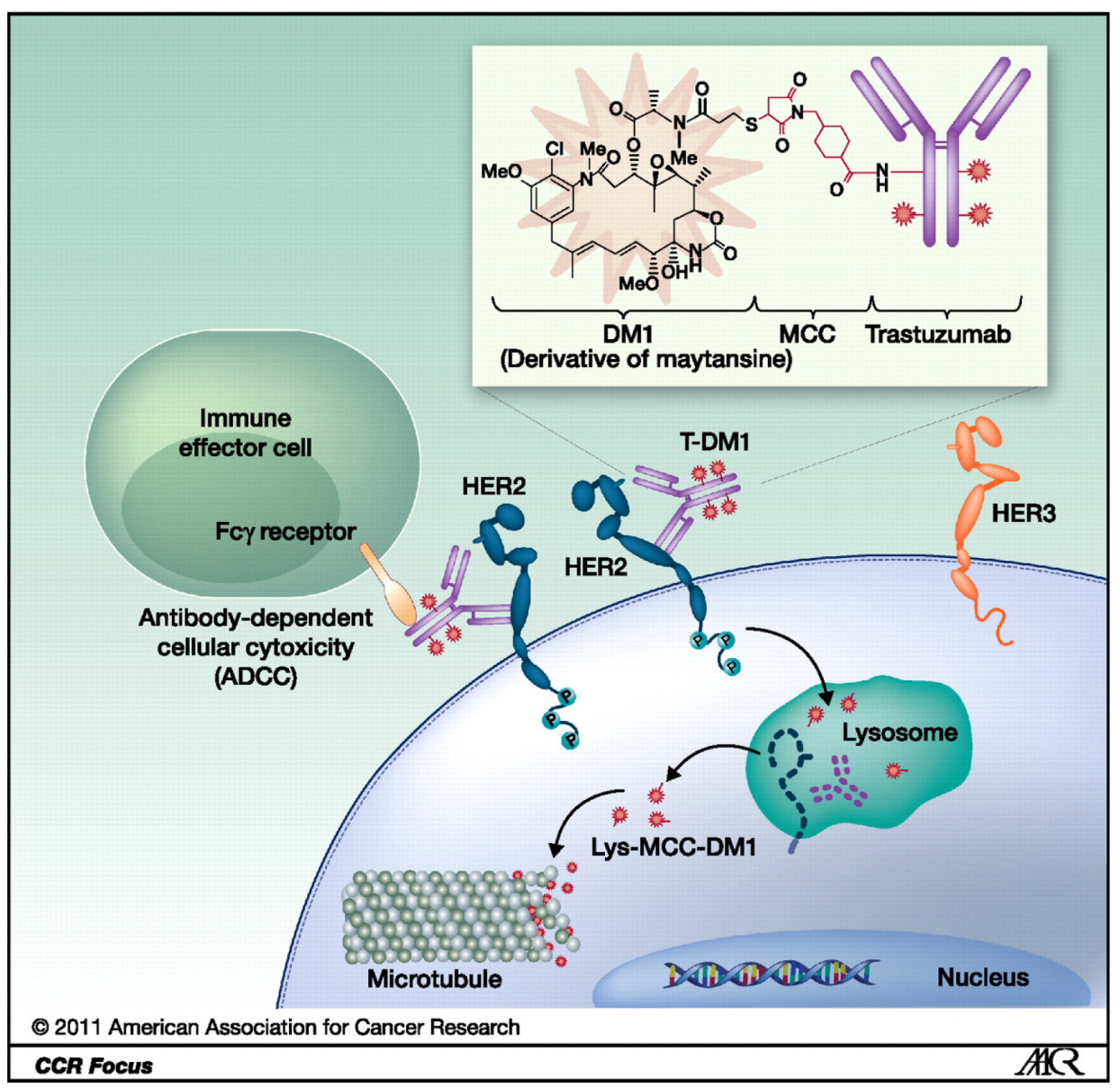

Figure I Structure of trastuzumab emtansine and mechanisms of action.

Notes: On binding of trastuzumab emtansine (T-DMI) to human growth factor receptor 2 (HER2), T-DMI is internalized and undergoes lysosomal degradation. This results in the release of DMI, which binds to tubulin, resulting in the suppression of microtubule dynamic instability and prevention of microtubule polymerization. T-DMI has also been shown to retain mechanisms of action of trastuzumab, including disruption of HER2 signal transduction and antibody-dependent cellular cytotoxicity (ADCC). Reprinted by permission from the American Association for Cancer Research: LoRusso PM, Weiss D, Guardino E, Girish S, Sliwkowski MX. Trastuzumab emtansine: a unique antibody-drug conjugate in development for human epidermal growth factor receptor 2-positive cancer. Clin Cancer Res. 20 I I; I7(20):6437-6447. DOI: I0.I I58/I078-0432.CCR-I I-0762. ${ }^{4}$

Abbreviation: MCC, 4-[N-maleimidomethyl] cyclohexane-I-carboxylate.

microtubule cytoskeleton and inhibits proplatelet production while also inhibiting megakaryocyte differentiation. ${ }^{18}$

\section{Completed clinical studies Phase I trials}

T-DM1 was first evaluated in a phase 1, open-label, multicenter, dose-escalation study (TDM3569g) in which patients with HER2+ MBC whose disease had progressed on prior trastuzumab-containing therapy were assigned to one of two dosing regimens: every 21 days or weekly. ${ }^{19,20}$ The three (q3) weekly dosing cohort was enrolled first. A total of 24 patients received escalating doses of intravenous T-DM1 starting at $0.3 \mathrm{mg} / \mathrm{kg}$ every 21 days. ${ }^{19}$ The median number of prior regimens received in the metastatic setting was four (range, one to eight), and $58 \%$ of patients had at least three distinct sites of metastatic disease. Dose-limiting grade 4 thrombocytopenia was observed in two of three patients treated with $4.8 \mathrm{mg} / \mathrm{kg}$, and the maximum tolerated dose (MTD) was identified as $3.6 \mathrm{mg} / \mathrm{kg}$. In this heavily pretreated patient population, six of 24 patients achieved a partial response, five of which were confirmed, yielding an overall objective response rate (ORR) of $21 \%$. A total of 15 patients were treated with $3.6 \mathrm{mg} / \mathrm{kg}$. The clinical benefit rate (objective response plus stable disease at 6 months) among the 15 patients who were treated at the MTD was $73 \%$. Of these 15 patients, nine had measurable disease, including four patients who had a confirmed partial response for an ORR of $44 \%$ (four of nine) in this group. The most commonly reported all-grade adverse events (AEs) were thrombocytopenia $(54.2 \%)$, elevated transaminases $(41.7 \%)$, fatigue $(37.5 \%)$, anemia (29.2\%), and nausea (25\%). Of note, no neuropathy was noted in the study. ${ }^{19}$

In the second part of this study, 28 patients with HER2+ MBC were treated with escalating doses of weekly T-DM1, starting at $1.2 \mathrm{mg} / \mathrm{kg}$. ${ }^{20}$ Two of three patients treated with $2.9 \mathrm{mg} / \mathrm{kg}$ developed grade $3 \mathrm{AEs}$ (one grade 3 aspartate ami- 
Table I Summary of published studies with T-DMI

\begin{tabular}{|c|c|c|c|c|}
\hline Trial name & Patients, $\mathbf{n}$ & Treatment groups & Results & Reference \\
\hline \multicolumn{5}{|l|}{ Phase I } \\
\hline \multicolumn{5}{|l|}{ TDM3569g } \\
\hline Cohort I & 24 & Dose escalation, T-DMI q3 weeks & ORR, $44 \% ; C B R, 73 \%$ & 17 \\
\hline Cohort 2 & 28 & Dose escalation, T-DMI ql week & ORR, $46.4 \%$ & 18 \\
\hline \multicolumn{5}{|l|}{ Phase 2} \\
\hline TDM4258g & 112 & T-DMI: $3.6 \mathrm{mg} / \mathrm{kg}$ IV q3 weeks & PFS, 4.6 months; ORR, $26 \%$ & 20 \\
\hline TDM4374g & 110 & T-DMI: $3.6 \mathrm{mg} / \mathrm{kg}$ IV q3 weeks & PFS, 7.3 months; ORR, 35\%; CBR, 48\% & 21 \\
\hline TDM4450g & 137 & T-DMI: $3.6 \mathrm{mg} / \mathrm{kg}$ IV versus docetaxel + trastuzumab & PFS, I 4.2 months; ORR, $64 \%$ & 22 \\
\hline TDM4373g & 64 & $\begin{array}{l}\text { T-DMI: } 3.6 \mathrm{mg} / \mathrm{kg} \text { IV + pertuzumab } 840 \mathrm{mg} \text { loading } \\
\text { dose followed by } 420 \mathrm{mg} \text { IV q3 weeks }\end{array}$ & PFS, 6.6 months; ORR, 57\% & 23 \\
\hline \multicolumn{5}{|l|}{ Phase 3} \\
\hline EMILIA & 991 & $\begin{array}{l}\text { T-DMI: } 3.6 \mathrm{mg} / \mathrm{kg} \text { IV q3 weeks versus capecitabine + } \\
\text { lapatinib }\end{array}$ & $\begin{array}{l}\text { OS, } 30.9 \text { months; PFS, } 9.6 \text { months; } \\
\text { ORR, } 44 \%\end{array}$ & 24 \\
\hline TH3RESA & 602 & T-DMI: 3 mg/kg IV q3 weeks versus physician choice & PFS, 6.2 months; ORR, $31 \%$ & 25 \\
\hline
\end{tabular}

Abbreviations: T-DMI, trastuzumab emtansine; qI, weekly; q3; every 3 weeks; ORR, objective response rate; CBR, clinical benefit rate defined as objective response plus stable disease lasting at least 6 months; IV, intravenous; PFS, progression-free survival; OS, overall survival; EMILIA, An Open-label Study of Trastuzumab Emtansine (T-DMI) vs Capecitabine + Lapatinib in Patients With HER2-positive Locally Advanced or Metastatic Breast Cancer; TH3RESA, A Study of Trastuzumab Emtansine in Comparison With Treatment of Physician's Choice in Patients With HER2-positive Breast Cancer Who Have Received at Least Two Prior Regimens of HER2-directed Therapy; HER2, human growth factor receptor 2 .

notransferase elevation and one grade 3 thrombocytopenia), thus establishing the MTD of the weekly regimen as $2.4 \mathrm{mg} / \mathrm{kg}$. Objective partial responses were seen in 13 of the 28 patients $(46.4 \%)$. A total of 16 patients were treated at the MTD, 15 of whom had measurable disease. The ORR in these 15 patients was $40 \%$ (six of 15 ). Similar to the q3 weekly dosing regimen, ${ }^{19}$ there was no grade 3 neuropathy observed, and the rate of grade $3 / 4$ AEs $(68 \%)$ was similar to that reported with the q3 weekly regimen $(50 \%) .{ }^{20}$ It is interesting to consider that the weekly regimen delivers twice as much T-DM1 and, based on these data, appears to have similar tolerability to the $\mathrm{q} 3$ weekly regimen. Although the ORR of all patients enrolled is higher with the weekly regimen, given the very small size of this study, a relative comparison of efficacy is not possible. Ongoing studies are evaluating the weekly regimen further. ${ }^{21} \mathrm{~A}$ summary of Phase I trials are provided in Table 1.

\section{Phase 2 trials}

TDM4258 was a proof-of-concept phase 2 study to evaluate the activity and safety of $\mathrm{q} 3$ weekly T-DM1 in patients with previously treated HER $2+\mathrm{MBC} .{ }^{22}$ In this single-group, open-label study, 112 heavily pretreated patients were enrolled. The median number of previous lines of therapy was 8 and included prior taxane (84\%), anthracycline (71\%), capecitabine (66\%), and carboplatin (42\%). Prior lapatinib was used in $60 \%$ of patients. The ORR was $25.9 \%$, and the median progression-free survival (PFS) was 5.3 months by independent review. Among patients previously treated with lapatinib ( $\mathrm{n}=66)$, the ORR was $24.2 \%$, and the median PFS was 4.2 months. The safety profile was similar to that reported in the phase 1 studies. Importantly, no patients developed grade $3 / 4$ congestive heart failure or left ventricular ejection fraction decline, and no patients came off study because of cardiac toxicity. HER2 status was evaluated centrally on tumors from 95 patients and was found to be positive in 74 . The ORR in those patients with confirmed HER2+ tumors was $33.8 \%$, and median PFS was 8.2 months. Interestingly, 21 patients had HER 2 normal tumors, and of those, only one patient had an objective response. ${ }^{22}$

A confirmatory, single-group phase 2 study (TDM4374g) of q3 weekly T-DM1 was conducted in 110 patients who had been previously exposed to trastuzumab, anthracyclines, taxanes, and capecitabine. ${ }^{23}$ In contrast to the TDM4258 study, ${ }^{22}$ this study required patients to have received prior lapatinib. The primary endpoint was independently assessed ORR. The ORR was $34.5 \%$ (38/110), clinical benefit rate was $48.2 \%$, and duration of response was 7.2 months. The median PFS was 6.9 months by independent review. The ORR was similar for patients who had documented progression on prior lapatinib, trastuzumab, and chemotherapy. Central confirmation of HER2 status was performed on archival tumor tissue from 95 patients. Of these patients, 15 were found to be HER2 normal, three of whom had an objective response (20\%). In contrast, $41 \%$ of patients with centrally confirmed HER2+ disease had an objective response. The most common all-grade AEs were fatigue, nausea, and thrombocytopenia, and the most frequent grade 3/4 AEs were thrombocytopenia, fatigue, and cellulitis. Elevation of aspartate aminotransferase occurred in $26 \%$, and of ALT in $14 \%$, of the patients. A total 
of nine patients $(8 \%)$ experienced grade 3 or higher hepatic event, including seven patients with grade 3 laboratory abnormalities, one patient with grade 3 liver toxicity, and one patient who had a fatal hepatic event. Thrombocytopenia was also observed in $38 \%$ of patients. However, hemorrhagic events were typically mild, with the exception of one grade 3 epistaxis. Similar to the TDM4258 study, ${ }^{22}$ no patients came off study because of cardiac toxicity. ${ }^{23}$

The first randomized study to evaluate T-DM1 was a phase 2 trial (TDM4450g) comparing q3 weekly T-DM1 to q3 weekly docetaxel plus trastuzumab in 137 patients with previously untreated HER2+ MBC. ${ }^{24}$ Patients in the control group received trastuzumab $(8 \mathrm{mg} / \mathrm{kg}$ intravenous loading dose, followed by $6 \mathrm{mg} / \mathrm{kg}$ thereafter) plus docetaxel ( 75 or $100 \mathrm{mg} / \mathrm{m}^{2}$, per investigator choice). The ORR (58\% in the control group versus $64 \%$ in the T-DM1 group; $P=0.458$ ) and clinical benefit rate were similar in the two treatment groups; however, T-DM1 was associated with a significant 5 month prolongation in median PFS (9.2 months versus 14.2 months; hazard ratio [HR], 0.59; 95\% confidence interval [CI], 0.36-0.97). The median duration of response was 9.5 months in the docetaxel/trastuzumab group and was not reached in the TDM-1 group. Importantly, there was a much lower rate of grade 3/4 AEs in the T-DM1 group (90.9\% versus $46.4 \%$ ), underscoring the considerable safety imparted by targeted delivery of chemotherapy using an ADC that has a stable linker. In fact, grade 4 AEs occurred in $58 \%$ of patients in the standard docetaxel/trastuzumab group compared with only $6 \%$ in the T-DM1 group. In the docetaxel/trastuzumab group, the most common toxicities were alopecia, neutropenia, diarrhea, and fatigue. More patients in the control group used colony-stimulating factors compared with in the T-DM1 group (44\% versus 6\%). In the TDM-1 group, the AEs consisted mostly of fatigue, nausea, elevated serum aspartate aminotransferase, fever, and headache. ${ }^{24}$ The lower rate of AEs in the T-DM1 group translated to an improvement in quality of life. The time to a decrease in 5 or more points in the Trial Outcome Index-Physical/Functional/Breast (TOI-PFB) score was significantly delayed in the T-DM1 group (3.5 months in the control group versus 7.5 months in the T-DM1 group; HR, 0.58; 95\% CI, 0.36-0.92; $P=0.022) .{ }^{24}$

The safety and activity of dual-HER2 (see Table 1) targeted therapy with T-DM1 plus another HER2-targeted monoclonal antibody, pertuzumab, was evaluated in a phase 2a trial, TDM4373g. ${ }^{25}$ In this study, 64 patients with HER2+ MBC (43 in the second-line or greater setting and 21 in the first-line setting) were treated with T-DM1 $(3.6 \mathrm{mg} / \mathrm{kg}) \mathrm{plus}$ pertuzumab (840 mg loading dose, followed by $420 \mathrm{mg}$ ) q3 weeks. Of the 21 first-line patients, $86 \%$ had received trastuzumab in the early-stage setting. The 43 patients who were previously treated for $\mathrm{MBC}$ had received a median of six prior lines of nonhormonal therapy. The ORR for the entire population was $41 \%$. In the first-line patients, the ORR was 57\% and in the advanced setting was 33\%. The median PFS was 6.6 months, with 7.7 months in the first-line and 5.5 months in the second-line patients and beyond. The most common grade 3 or higher AEs were thrombocytopenia (13\%), fatigue $(11 \%)$, and transaminase elevations $(9 \%){ }^{25}$

\section{Phase 3 trials}

In the landmark phase 3 EMILIA trial, 991 patients with metastatic or locally advanced trastuzumab-pretreated HER2+ disease were randomly assigned to receive either lapatinib (1,250 mg daily continuously) plus capecitabine $\left(1,000 \mathrm{mg} / \mathrm{m}^{2}\right.$ by mouth twice a day, 14 days on, 7 days off) or T-DM1 q3 weeks. ${ }^{26}$ The primary endpoints were PFS, OS, and safety. The median PFS was 9.6 months in the T-DM1 group versus 6.4 months in the lapatinib plus capecitabine group (HR, 0.65; 95\% CI, 0.55-0.77; $P<0.001)$. At the second interim analysis, median OS crossed the stopping boundary for efficacy and was increased by 5 months in the T-DM1 group (30.9 months versus 25.1 months; HR, 0.68; 95\% CI, 0.55-0.85; $P<0.001)$. Secondary endpoints including ORR (43.6\% versus $30.8 \%$; $P<0.001)$ and median duration of response (12.6 months versus 6.5 months) favored T-DM1. In addition, the median time to decrease of 5 points or more in the Functional Assessment of Cancer Therapy-Breast (FACT-B TOI) score was delayed in the T-DM1-treated patients (7.1 months versus 4.6 months; HR, 0.80; 95\% CI, 0.67-0.95; $P=0.012$ ).

Grade 3/4 AEs were less frequent in the T-DM1-treated patients compared with the control group patients $(40.8 \%$ versus $57.0 \%$ ). The most common grade $3 / 4$ AEs in the T-DM1-treated patients were thrombocytopenia and increased liver transaminases, and the most common grade 3/4 AEs in control group patients were diarrhea and palmar-plantar erythrodysesthesia. Of note, the rate of cardiac dysfunction was similar in both groups, with a total of $1.7 \%$ of patients in the T-DM1 group and $1.6 \%$ of patients in lapatinib plus capecitabine group having an ejection fraction that was below $50 \%$, or at least $15 \%$ below baseline. Grade 3 congestive heart failure was seen in only one patient in the T-DM1 group. ${ }^{26}$

The activity of T-DM1 in the lapatinib-pretreated HER2+ MBC setting was explored in the TH3RESA study. ${ }^{27}$ In this second phase 3 trial of T-DM1, patients who had received at least two HER2-targeted therapies for advanced disease, including both trastuzumab and lapatinib, were randomized 
in an open-label fashion to receive T-DM1 or treatment of physicians choice (TPC). A total of 602 patients were randomized in a 2:1 ratio to T-DM1 (3.6 mg/kg q21 days) or TPC. Stratification was based on region of the world, number of prior regimens, and presence or absence of visceral disease. Coprimary endpoints were investigator-assessed PFS and $\mathrm{OS}$ in the intent-to-treat population. Patients had received a median of four prior regimens for MBC. In the TPC group, $83 \%$ of the patients received HER2-targeted therapy as part of their regimen, and $17 \%$ received single-agent chemotherapy alone. The median PFS was significantly longer in the T-DM1 group compared with in the TPC group (6.2 months versus 3.3 months; HR, 0.528; 95\% CI, 0.442-0.661; $P<0.0001$ ). There was a trend for improved survival with T-DM1, but the stopping boundary was not crossed at the first interim analysis. The ORR was also significantly improved in patients with measurable disease treated with T-DM1 (31\% versus 9\%, 95\% CI, 16.2-29.2; $P<0.0001)$. In terms of grade 3 or greater toxicities, thrombocytopenia was the only AE reported more commonly in the T-DM1 group. Overall, 18\% of patients in the T-DM1 group and $21 \%$ of patients in the TPC group experienced a significant adverse event. ${ }^{27}$

The consistent PFS and ORR benefit seen in these two published phase 3 trials confirm that T-DM1 is a highly active and better-tolerated agent for patients with HER2+ MBC after the first-line setting (Table 1). Unfortunately, patients eventually experience disease progression on T-DM1; hence, discovering molecular markers that can predict response or resistance to this therapy remains a high priority.

\section{Predictors of response or resistance to T-DMI HER2 expression levels}

HER2 expression levels do appear to influence the efficacy outcomes associated with T-DM1. In exploratory analyses of several phase 2 and phase 4 studies, HER2 expression levels were measured on archived tumor samples by quantitative reverse transcription polymerase chain reaction. Patients were grouped according to whether their HER2 expression was greater than or less than the median. Of 50 patients included in the TDM4258g analysis, the median PFS was not reached (95\% CI, 4.6 months- non estimable [NE]) for those with median HER 2 expression or worse and was 4.2 months (95\% CI, 2.7-6.8 months) for those patients with lower than median HER2 expression. ${ }^{22}$ In the TDM4374 trial, patients in whom HER2 expression was at least the median had a higher ORR of $42 \%$ and a PFS of 8.0 months compared with those in whom HER2 expression level was below the median (ORR, 38\%;
PFS, 6.2 months). ${ }^{23}$ Improved ORR and PFS were also reported in pertuzumab plus T-DM1-treated patients with tumor HER2 mRNA levels at the median or higher in the TDM4373g study. ${ }^{25}$ A similar analysis was undertaken in the TDM4450 study, in which HER2 mRNA levels were available for 116/137 patients: 61 in the control group and 55 in the T-DM1 group. ${ }^{28}$ In this randomized study, the relative risk of progressive disease in the overall population was reduced by $41 \%$ with T-DM1 compared with chemotherapy plus trastuzumab. This effect was even higher in patients with HER2 mRNA levels at least the median $(61 \%$ relative risk reduction favoring the T-DM1 group; HR, 0.39; 95\% CI, 0.18-0.85) compared with those with HER 2 mRNA levels less than the median ( $15 \%$ relative risk reduction favoring the T-DM1 group; $\mathrm{HR}, 0.85 ; 95 \% \mathrm{CI}$, 0.44-1.67). The median PFS was not reached in the T-DM1 group in patients with HER 2 mRNA at least median and was 10.6 months for those with HER2 mRNA less than median. In the control group (trastuzumab/docetaxel), median PFS was not significantly different (8.8 months versus 9.8 months with HER2 mRNA at the median or higher versus lower than median, respectively). ${ }^{28}$ Biomarker analysis of the EMILIA trial further confirmed this observation. Patients with tumors expressing HER2 levels greater than median had an OS of 34.1 months and a PFS of 10.6 months compared with 26.5 months and 8.2 months, respectively, for patients with HER2 levels less than or equal to the median. ${ }^{29}$ The consistent observation that HER2 expression influences response to T-DM1 is intriguing, but further analyses including prospective studies are needed to confirm the threshold of HER2 expression required for a response to this therapy.

\section{Drug efflux pumps}

$\mathrm{P}$ glycoprotein I (also known as MDR1) is the best-known transporter that mediates the efflux of toxins and drugs from the cell. MDR1 expression has been associated with poor response to chemotherapy in malignancies..$^{30}$ In one study, a T-DM1-resistant cell line was shown to have upregulation of the MDR transporters. ${ }^{31}$ Another study evaluated two cell lines (JIMT1 and MDA-MB-361) that had conditioned resistance to a trastuzumab-maytanisinoid conjugate (JIMT-TM and 361-TM) and showed that JIMT-TM resistance may be related to downregulation in HER2 expression, as well as increased expression in proteins related to posttranslational modification and endosomal proteins; however, drug efflux proteins were not altered. In contrast, in 361-TM cells, an increase in ABCC (MRP1) was seen without any changes in ABCB1 (MDR1). ${ }^{32}$ Additional studies are needed to assess the importance of MDR in mitigating clinical resistance to T-DM1. 


\section{PIK3CA mutations}

At least one mechanism involved in the oncogenic function of HER 2 is activation of the phosphoinositide 3-kinase (PI3K)-Akt signaling pathway. Activating mutations in the catalytic subunit of the PI3K (PIK3CA) can result in increased activity of this pathway. ${ }^{33}$ It is estimated that $20 \%-40 \%$ of HER2+ breast cancers have activating mutations in PIK 3 CA. ${ }^{34}$ Preclinical work has demonstrated that HER2+/PIK3CA mutant tumors are resistant to trastuzumab alone or in combination with lapatinib or pertuzumab. ${ }^{35}$ Exploratory analyses from two recent trials confirm this laboratory phenomenon. The NeoSphere study was a randomized phase 2 trial that demonstrated that dual HER2-targeting with neoadjuvant pertuzumab, trastuzumab, and docetaxel was associated with a higher pathological complete response ( $\mathrm{pCR}$ ) rate of $45.8 \%$ compared with three other combinations (docetaxel and trastuzumab [pCR, 29\%], trastuzumab and pertuzumab [pCR, 16.8\%], and docetaxel and pertuzumab [pCR, 24.0\%]). The rate of $\mathrm{pCR}$ was lower in all four group in patients whose tumors bore PIK3CA mutations (24\%) compared with patients without PIK3CA mutations (35\%). ${ }^{36}$ Biomarker analysis of the NeoALTTO trial, another neoadjuvant study in HER2+ breast cancer that evaluated lapatinib and/or trastuzumab plus paclitaxel, also confirms this observation. ${ }^{37}$ Patients whose tumors had PIK3CA mutations had a lower pCR rate, with the most notable difference in the group containing both lapatinib and the trastuzumab group (55.8\% without mutations versus $28.6 \%$ with mutations) ${ }^{38}$ Interestingly, an exploratory analysis on the EMILIA trial confirms that PIK3CA mutations were associated with a decrease in PFS in the capecitabine/lapatinib group (6.4 months wild-type versus 4.3 months mutated); however, the presence of PIK3CA mutations did not correlate with outcome in the T-DM1 group (9.8 months wild-type versus 10.9 months mutated).$^{29}$ Thus, although the presence of PIK3CA mutations may be prognostic for poorer outcome in patients treated with trastuzumab, pertuzumab, or lapatinib, this marker does not appear to be prognostic of outcome in patients treated with T-DM1.

\section{Neuregulin-HER3 signaling}

Recent translational work provides some evidence that resistance to T-DM1 may in part be mediated by neuregulin and HER3 signaling. Neuregulin-1 is a ligand that binds to HER3, inducing a conformational change allowing HER3 to dimerize with HER2. HER2:HER3 dimers are highly potent activators of the PI3K pathway. ${ }^{39}$ Pertuzumab, a monoclonal HER2-targeted antibody, binds to and blocks HER2 at the HER3-binding domain, thus abrogating PI3K signaling. Preclinical work led by Phillips and colleagues demonstrated that in the presence of neuregulin, enhanced antiproliferative effects were seen when T-DM1 was combined with pertuzumab compared with T-DM1 alone in three cell lines (Calu-3 lung, BT-474, and SK-BR-3). There was no combination effect seen in KPL-4 cells that harbor PIK3CA-activating mutations, however, even in the presence of neuregulin. ${ }^{40}$ These intriguing preclinical results led to the phase $1 \mathrm{~b} / 2$ TDM4373g clinical trial evaluating pertuzumab plus T-DM1 in HER2+ MBC (see "Phase 2 trials" section). ${ }^{25}$

\section{Future directions}

Although the benefit of T-DM1 in patients with pretreated, locally advanced, or metastatic HER $2+$ breast cancer is clear, additional studies are needed to evaluate the benefit of T-DM1 in combination with other agents as well as its role in early-stage HER2+ disease. One ongoing trial, MARIANNE (A Study of Trastuzumab Emtansine [T-DM1] Plus Pertuzumab/Pertuzumab Placebo Versus Trastuzumab [Herceptin] Plus a Taxane in Patients With Metastatic Breast Cancer; NCT01120184), is a three-group study in previously untreated locally advanced or metastatic HER2+ breast cancer that compares trastuzumab plus taxane, T-DM1 plus pertuzumab, and T-DM1 plus placebo. All patients are treated until disease progression with primary endpoint of PFS and a secondary endpoint of OS, as well as biomarker studies. This study has completed accrual with results anticipated in late 2014. The TDM4652g trial (NCT00951665) is a phase $1 \mathrm{~b}$ trial exploring the combination of T-DM1, paclitaxel \pm pertuzumab in previously treated patients with metastatic breast cancer. Finally, the BP22572 trial (NCT00934856) is a phase $1 \mathrm{~b} / 2 \mathrm{a}$ trial to explore combination of T-DM1 with docetaxel with or without pertuzumab in previously treated HER2+ MBC.

The activity and safety of T-DM1 in early breast cancer is being explored by several other trials. The ATEMPT (T-DM1 vs Paclitaxel/Trastuzumab for Breast, NCT01853748) trial is a phase 2 study in which patients with stage 1 HER2+ breast cancer are randomized to T-DM1 every 3 weeks for 17 weeks or to a combination of trastuzumab and paclitaxel weekly for 12 weeks followed by maintenance trastuzumab to complete 1 year. The primary endpoint of the trial is disease-free survival, and the secondary endpoint is OS. The randomized, open-label phase 3 KAITLIN (A Study of Kadcyla [Trastuzumab Emtansine] Plus Perjeta [Pertuzumab] Following Anthracyclines in Comparison With Herceptin [Trastuzumab] Plus Perjeta and a Taxane Following Anthracyclines as Adjuvant Therapy in Patients With Operable HER2-Positive Primary Breast Cancer, NCT01966471) trial is comparing the combination of trastuzumab and pertuzumab plus 
a taxane versus T-DM1 plus pertuzumab after completion of adjuvant anthracycline-containing chemotherapy. The role of T-DM1 in the neoadjuvant setting is being explored in the KRISTINE (A Study Comparing Kadcyla Plus Perjeta Treatment to Chemotherapy Combined With Herceptin Plus Perjeta in Patients With HER2-Positive Breast Cancer, NCT02131064) trial. In this open-label phase 2 trial, patients are randomized to either six cycles of q3 weekly docetaxel, carboplatin, trastuzumab, and pertuzumab or T-DM1 and pertuzumab before definitive surgery. Another neoadjuvant study is ADAPT (A Prospective, Randomized Multicenter, Open-label Comparison of Preoperative Trastuzumab Emtansine [T-DM1] With or Without Standard Endocrine Therapy vs Trastuzumab With Standard Endocrine Therapy Given for Twelve Weeks in

Table 2 Ongoing clinical trials with T-DMI

\begin{tabular}{|c|c|c|c|c|}
\hline Trial name & Phase & Patient population & $\begin{array}{l}\text { Number to } \\
\text { be enrolled }\end{array}$ & Treatment groups \\
\hline MARIANNE (NCTOII20I84) & 3 & First-line HER2+ MBC & $\mathrm{I}, 092$ & $\begin{array}{l}\text { T-DMI + placebo versus T-DMI + pertuzumab versus } \\
\text { trastuzumab + a taxane }\end{array}$ \\
\hline TDM4652g (NCT0095I665) & Ib & Previously treated HER2+ MBC & 107 & T-DMI + paclitaxel \pm pertuzumab \\
\hline BP22572 (NCT00934856) & $\mathrm{Ib} / 2 \mathrm{a}$ & $\begin{array}{l}\text { Previously treated } \\
\text { HER2+ MBC }\end{array}$ & 99 & T-DMI + docetaxel \pm pertuzumab \\
\hline KAITLIN (NCT0I96647I) & 3 & Adjuvant & 2,500 & $\begin{array}{l}\mathrm{AC} / \mathrm{FEC} \rightarrow \mathrm{T} \text {-DMI/pertuzumab versus } \\
\mathrm{AC} / \mathrm{FEC} \rightarrow \text { trastuzumab/pertuzumab/taxane }\end{array}$ \\
\hline ATEMPT (NCT0I853748) & 2 & Stage I adjuvant & 500 & $\begin{array}{l}\text { T-DMI } \times I \text { year versus paclitaxel/trastuzumab } \times 12 \text { weeks, } \\
\text { followed by trastuzumab maintenance to complete I year }\end{array}$ \\
\hline KRISTINE (NCT02I3I064) & 2 & Neoadjuvant & 432 & $\begin{array}{l}\text { T-DMI/pertuzumab versus taxotere/carboplatin/ } \\
\text { trastuzumab/pertuzumab }\end{array}$ \\
\hline ADAPT (NCT0I745965) & 2 & Neoadjuvant & 380 & $\begin{array}{l}\text { T-DMI } \pm \text { endocrine therapy versus trastuzumab }+ \\
\text { endocrine therapy for } 12 \text { weeks before surgery }\end{array}$ \\
\hline KATHERINE (NCTOI772472) & 3 & $\begin{array}{l}\text { Residual disease after } \\
\text { neoadjuvant therapy }\end{array}$ & $\mathrm{I}, 484$ & $\begin{array}{l}\text { T-DMI versus trastuzumab maintenance for } 14 \text { cycles } \\
\text { after surgery }\end{array}$ \\
\hline $\begin{array}{l}\text { Trastuzumab and Pertuzumab } \\
\text { followed by T-DMI in MBC } \\
\text { (NCT0I835236) }\end{array}$ & 2 & First-line HER2+ MBC & 208 & $\begin{array}{l}\text { Trastuzumab and pertuzumab (no chemotherapy) in } \\
\text { first-line MBC followed by T-DMI in second-line MBC in } \\
\text { the event of progression versus trastuzumab/pertuzumab/ } \\
\text { paclitaxel or vinorelbine in first-line MBC followed by } \\
\text { T-DMI in second-line MBC in event of progression }\end{array}$ \\
\hline $\begin{array}{l}\text { BYL7I9 + T-DMI in HER2(+) } \\
\text { Metastatic Breast Cancer } \\
\text { Pts Who Progress on Prior } \\
\text { Trastuzumab and Taxane Tx } \\
\text { (NCT020380I0) }\end{array}$ & I & $\begin{array}{l}\text { Previously treated HER2+ } \\
\text { MBC with progression on prior } \\
\text { trastuzumab and taxane-based } \\
\text { therapy }\end{array}$ & 28 & $\begin{array}{l}\text { BYL7I9 (PI3K inhibitor) orally daily on days I-2I and } \\
\text { T-DMI every } 3 \text { weeks }\end{array}$ \\
\hline $\begin{array}{l}\text { GDC-094 I administered orally } \\
\text { to patients with Trastuzumab- } \\
\text { DMI or trastuzumab } \\
\text { (NCT00928330) }\end{array}$ & I & $\begin{array}{l}\text { Previously treated HER2+ } \\
\text { MBC who progressed on } \\
\text { trastuzumab-based therapy }\end{array}$ & 57 & $\begin{array}{l}\text { Oral GDC-094I (PI3K inhibitor) daily and T-DMI or } \\
\text { trastuzumab }\end{array}$ \\
\hline STELA (NCT02073916) & I & $\begin{array}{l}\text { Previously treated MBC with } \\
\text { at least two prior therapies }\end{array}$ & 18 & $\begin{array}{l}\text { T-DMI intravenously every } 3 \text { weeks in combination with } \\
\text { lapatinib daily plus abraxane intravenously weekly }\end{array}$ \\
\hline $\begin{array}{l}\text { ONT380 + TDMI } \\
(\text { NCT0I98350I) }\end{array}$ & I & $\begin{array}{l}\text { Previously treated HER2+ MBC } \\
\text { with taxane and trastuzumab }\end{array}$ & 48 & ONT380 orally twice a day with T-DMI intravenously \\
\hline $\begin{array}{l}\text { Phase Ib Study of } \\
\text { PD-033299I in Combination } \\
\text { With T-DMI (NCT0I976I69) }\end{array}$ & I & $\begin{array}{l}\text { Previously treated MBC with } \\
\text { HER2+ and RB proficient tumor }\end{array}$ & 17 & $\begin{array}{l}\text { T-DMI intravenously on day I with PD-033299I } \\
\text { (palbociclib) on days 5-I8 of every } 21 \text {-day cycle }\end{array}$ \\
\hline
\end{tabular}

Abbreviations: MARIANNE, A Study of Trastuzumab Emtansine (T-DMI) Plus Pertuzumab/Pertuzumab Placebo Versus Trastuzumab [Herceptin] Plus a Taxane in Patients With Metastatic Breast Cancer; HER, human growth factor receptor; MBC, metastatic breast cancer; TDM4652g, A Study of Trastuzumab Emtansine, Paclitaxel, and Pertuzumab in Patients With HER2-Positive, Locally Advanced or Metastatic Breast Cancer; T-DMI, trastuzumab emtansine; BP22572, A Study of Trastuzumab Emtansine (T-DMI) in Combination With Docetaxel, and Potentially Pertuzumab, in Patients With Advanced Breast Cancer; KAITLIN, A Study of Kadcyla (Trastuzumab Emtansine) Plus Perjeta (Pertuzumab) Following Anthracyclines in Comparison With Herceptin (Trastuzumab) Plus Perjeta and a Taxane Following Anthracyclines as Adjuvant Therapy in Patients With Operable HER2-Positive Primary Breast Cancer; AC/FEC, adriamycin, cyclophosphamide/5-fluorouracil, epirubicin, and cyclophosphamide; ATEMPT, T-DMI vs Paclitaxel/Trastuzumab for Breast; KRISTINE, A Study Comparing Kadcyla Plus Perjeta Treatment to Chemotherapy Combined With Herceptin Plus Perjeta in Patients With HER2-Positive Breast Cancer; ADAPT, A Prospective, Randomized Multicenter, Open-label Comparison of Preoperative Trastuzumab Emtansine (T-DMI) With or Without Standard Endocrine Therapy vs Trastuzumab With Standard Endocrine Therapy Given for Twelve Weeks in Patients With Operable HER2+/HR+ Breast Cancer Within the ADAPT Protocol; KATHERINE, A Study of Trastuzumab Emtansine Versus Trastuzumab as Adjuvant Therapy in Patients With HER2-Positive Breast Cancer Who Have Residual Tumor in the Breast or Axillary Lymph Nodes Following Preoperative Therapy; GDC-094I administered orally to patients with Trastuzumab-DMI or trastuzumab, Trastuzumab and Trastuzumab-MCC-DMI Administered Intravenously and GDC-094I Administered Orally to Patients With HER2-Positive Metastatic Breast Cancer Who Have Progressed on Previous Trastuzumab-Based Therapy; STELA, TDMI With Abraxane and Lapatinib for Metastatic HER2 Positive Breast Cancer; ONT380 + TDMI, A Phase Ib Study of ONT-380 Combined With Ado-trastuzumab Emtansine (T-DMI) in Patients With HER2+ Breast Cancer. 
Patients With Operable HER2+/HR+ Breast Cancer Within the ADAPT Protocol, NCT01745965), a randomized trial evaluating T-DM1 (with or without endocrine therapy) versus trastuzumab plus endocrine therapy for 12 weeks before definitive surgery in HER2+/hormone receptor-positive breast cancer. The KATHERINE (A Study of Trastuzumab Emtansine Versus Trastuzumab as Adjuvant Therapy in Patients With HER2-Positive Breast Cancer Who Have Residual Tumor in the Breast or Axillary Lymph Nodes Following Preoperative Therapy, NCT01772472) trial will enroll patients who had residual disease at the time of definitive surgery after standard neoadjuvant trastuzumab-based therapy to either T-DM1 or trastuzumab maintenance for 14 cycles after surgery.

In addition to the abovementioned trials, studies are ongoing that will evaluate the combination of T-DM1 with other biologically targeted therapies. The BYL719 + T-DM1 trial (BYL719 + T-DM1 in HER2[+] Metastatic Breast Cancer Pts Who Progress on Prior Trastuzumab and Taxane Tx, NCT02038010) is a phase 1 trial exploring the safety of combination of BYL719, a PI3K inhibitor with T-DM1 in patients whose metastatic disease has progressed on prior trastuzumab and taxane-based therapy. Another PI3K inhibitor, GDC-0941 is also being evaluated in a phase 1 study (GDC-0941 + TDM1 or Trastuzumab, Trastuzumab and Trastuzumab-MCC-DM1 Administered Intravenously and GDC-0941 Administered Orally to Patients With HER2-Positive Metastatic Breast Cancer Who Have Progressed on Previous Trastuzumab-Based Therapy, NCT00928330) in combination with trastuzumab or T-DM1 for patients whose disease progressed on previous trastuzumabbased therapy. The STELA (TDM1 With Abraxane and Lapatinib for Metastatic HER2 Positive Breast Cancer, NCT02073916) trial is an open-label study to assess the tolerability and safety of combination of T-DM1 to lapatinib and $n a b$-paclitaxel in patients with HER2+ breast cancer who have received at least two prior therapies for their malignant disease. Dual HER2 blockade with the novel small molecule inhibitor of HER2, ONT-380 (A Phase 1b Study of ONT-380 Combined With Ado-trastuzumab Emtansine [T-DM1] in Patients With HER2+ Breast Cancer), along with T-DM1 is being evaluated in a small phase 1 study (NCT01983501) that will enroll 48 patients. Finally, the potential benefit of CDK4/6 inhibition in combination with T-DM1 will be evaluated in a phase 1 study of PD-0332991 along with T-DM1 (NCT01976169). In this study patients, will receive T-DM1 on day 1 followed by PD-0332991 orally on days 5-18 of every 21-day cycle. The ongoing clinical trials have been summarized in Table 2. Collectively, these trials will help not only address the role T-DM1 may have in early breast cancer but also elucidate the benefit of T-DM1 in combination with chemotherapy as well as other targeted therapies such as pertuzumab.

\section{Conclusion}

T-DM1 is the first example of a successfully developed ADC for the treatment of breast cancer. The collective published studies in phase 1 and 2, as well as the results of two large randomized phase 3 trials, EMILIA and TH3RESA, have demonstrated that T-DM1 is an efficacious and safe therapeutic intervention for HER2+ metastatic breast cancer. In spite of its success, disease progression on T-DM1 does occur; therefore, greater understanding of pathways of resistance is crucial in improving the efficacy. Ongoing studies will evaluate combining T-DM1 with other targeted therapies and using T-DM1 in the early setting.

\section{Disclosure}

$\mathrm{SAH}$ has received contracted funding from Genentech/ Roche (paid to the University of California, Los Angeles) for research and education and has had travel reimbursed for speaking at international conferences. SS has received honoraria from Genentech/Roche for speaker programs. OO reports no conflicts of interest in this work.

\section{References}

1. Slamon DJ, Clark GM, Wong SG, Levin WJ, Ullrich A, McGuire WL. Human breast cancer: correlation of relapse and survival with amplification of the HER-2/neu oncogene. Science. 1987;235(4785): 177-182.

2. Slamon DJ, Leyland-Jones B, Shak S, et al. Use of chemotherapy plus a monoclonal antibody against HER2 for metastatic breast cancer that overexpresses HER2. N Engl J Med. 2001;344(11):783-792.

3. Romond EH, Perez EA, Bryant J, et al. Trastuzumab plus adjuvant chemotherapy for operable HER2-positive breast cancer. NEngl J Med. 2005;353(16):1673-1684.

4. Piccart-Gebhart MJ, Procter M, Leyland-Jones B, et al; Herceptin Adjuvant (HERA) Trial Study Team. Trastuzumab after adjuvant chemotherapy in HER2-positive breast cancer. N Engl J Med. 2005;353(16): 1659-1672.

5. Slamon D, Eiermann W, Robert N, et al; Breast Cancer International Research Group. Adjuvant trastuzumab in HER2-positive breast cancer. N Engl J Med. 2011;365(14):1273-1283.

6. Dawood S, Broglio K, Esteva FJ, et al. Defining prognosis for women with breast cancer and CNS metastases by HER2 status. Ann Oncol. 2008;19(7):1242-1248.

7. Musolino A, Ciccolallo L, Panebianco M, et al. Multifactorial central nervous system recurrence susceptibility in patients with HER2-positive breast cancer: epidemiological and clinical data from a population-based cancer registry study. Cancer. 2011;117(9):1837-1846.

8. Geyer CE, Forster J, Lindquist D, et al. Lapatinib plus capecitabine for HER2-positive advanced breast cancer. N Engl J Med. 2006;355(26): 2733-2743.

9. Cameron D, Casey M, Press M, et al. A phase III randomized comparison of lapatinib plus capecitabine versus capecitabine alone in women with advanced breast cancer that has progressed on trastuzumab: updated efficacy and biomarker analyses. Breast Cancer Res Treat. 2008;112(3):533-543.

10. Hurvitz SA, Hu Y, O’Brien N, Finn RS. Current approaches and future directions in the treatment of HER2-positive breast cancer. Cancer Treat Rev. 2013;39(3):219-229.

11. Junttila TT, Li G, Parsons K, Phillips GL, Sliwkowski MX. TrastuzumabDM1 (T-DM1) retains all the mechanisms of action of trastuzumab and efficiently inhibits growth of lapatinib insensitive breast cancer. Breast Cancer Res Treat. 2011;128(2):347-356. 
12. Lewis Phillips GD, Li G, Dugger DL, et al. Targeting HER2-positive breast cancer with trastuzumab-DM1, an antibody-cytotoxic drug conjugate. Cancer Res. 2008;68(22):9280-9290.

13. Austin CD, Wen X, Gazzard L, Nelson C, Scheller RH, Scales SJ. Oxidizing potential of endosomes and lysosomes limits intracellular cleavage of disulfide-based antibody-drug conjugates. Proc Natl Acad Sci U S A. 2005;102(50):17987-17992.

14. Lewis Phillips GD, Li G, Dugger DL, et al. Targeting HER2-positive breast cancer with trastuzumab-DM1, an antibody-cytotoxic drug conjugate. Cancer Res. 2008;68(22):9280-9290.

15. Erickson HK, Park PU, Widdison WC, et al. Antibody-maytansinoid conjugates are activated in targeted cancer cells by lysosomal degradation and linker-dependent intracellular processing. Cancer Res. 2006;66(8):4426-4433.

16. Girish S, Gupta M, Wang B, et al. Clinical pharmacology of trastuzumab emtansine (T-DM1): an antibody-drug conjugate in development for the treatment of HER2-positive cancer. Cancer Chemother Pharmacol. 2012;69(5):1229-1240

17. Mahapatra K, Darbonne W, Bumbaca D, et al. T-DM1-induced thrombocytopenia results from impaired platelet production in a HER2independent manner. Mol Cancer Ther. 2011;10(Suppl 1):A135.

18. Thon JN, Devine MT, Jurak Begonja A, Tibbitts J, Italiano JE Jr. High-content live-cell imaging assay used to establish mechanism of trastuzumab emtansine (T-DM1) - mediated inhibition of platelet production. Blood. 2012;120(10):1975-1984.

19. Krop IE, Beeram M, Modi S, et al. Phase I study of trastuzumab-DM1, an HER2 antibody-drug conjugate, given every 3 weeks to patients with HER2positive metastatic breast cancer. J Clin Oncol. 2010;28(16): 2698-2704.

20. Beeram M, Krop IE, Burris HA, et al. A phase 1 study of weekly dosing of trastuzumab emtansine (T-DM1) in patients with advanced human epidermal growth factor 2-positive breast cancer. Cancer. 2012;118(23): 5733-5740.

21. Modi SEA, LoRusso P, Samant M, Guardino E, Althaus B, Krop IE. Results from a phase Ib study of trastuzumab emtansine (T-DM1), paclitaxel $(\mathrm{T})$, and pertuzumab $(\mathrm{P})$ in patients with HER2-positive metastatic breast cancer (MBC) previously treated with trastuzumab. In: 2012 Annual Meeting of the American Society of Clinical Oncology; June 1-5, 2012; Chicago, Illinois. Abstract 528.

22. Burris HA III, Rugo HS, Vukelja SJ, et al. Phase II study of the antibody drug conjugate trastuzumab-DM1 for the treatment of human epidermal growth factor receptor 2 (HER2)-positive breast cancer after prior HER2-directed therapy. J Clin Oncol. 2011;29(4):398-405.

23. Krop IE, LoRusso P, Miller KD, et al. A phase II study of trastuzumab emtansine in patients with human epidermal growth factor receptor 2-positive metastatic breast cancer who were previously treated with trastuzumab, lapatinib, an anthracycline, a taxane, and capecitabine. J Clin Oncol. 2012;30(26):3234-3241.

24. Hurvitz SA, Dirix L, Kocsis J, et al. Phase II randomized study of trastuzumab emtansine versus trastuzumab plus docetaxel in patients with human epidermal growth factor receptor 2-positive metastatic breast cancer. J Clin Oncol. 2013;31(9):1157-1163.

25. Miller KD, Diéras V, Harbeck N, et al. Phase IIa trial of trastuzumab emtansine with pertuzumab for patients with human epidermal growth factor receptor 2-positive, locally advanced, or metastatic breast cancer. J Clin Oncol. 2014;32(14):1437-1444.
26. Verma S, Miles D, Gianni L, et al; EMILIA Study Group. Trastuzumab emtansine for HER2-positive advanced breast cancer. $N$ Engl $\mathrm{J}$ Med. 2012;367(19):1783-1791.

27. Krop IE, Kim SB, González-Martín A, et al; TH3RESA study collaborators. Trastuzumab emtansine versus treatment of physician's choice for pretreated HER2-positive advanced breast cancer (TH3RESA): a randomised, open-label, phase 3 trial. Lancet Oncol. 2014;15(7):689-699.

28. Perez EA, Hurvitz SA, Amler LC, et al. Relationship between HER2 expression and efficacy with first-line trastuzumab emtansine compared with trastuzumab plus docetaxel in TDM4450g: a randomized phase II study of patients with previously untreated HER2-positive metastatic breast cancer. Breast Cancer Res. 2014;16(3):R50.

29. Baselga J, Verma S, Ro J, et al. Relationship between tumor biomarkers (BM) and efficacy in EMILIA, a phase III study of trastuzumab emtansine (T-DM1) in HER2-positive metastatic breast cancer (MBC). Cancer Res. 2013;73(Suppl 8):LB-63.

30. Takara K, Sakaeda T, Okumura K. An update on overcoming MDR1mediated multidrug resistance in cancer chemotherapy. Curr Pharm Des. 2006;12(3):273-286.

31. Li G, Fields CT, Parsons KL, Guo J, Lewis Phillips GD. TrastuzumabDM1: mechanisms of action and mechanisms of resistance. Eur $J$ Cancer. 2010;8(7):73.

32. Tan X, Jin G, Myers J, et al. Tumor cells selected for resistance to an antibody-drug conjugate retain sensitivity to ADCs with modified linkers and payloads. Cancer Res. 2013;73(Suppl 8):4629.

33. Engelman JA. Targeting PI3K signalling in cancer: opportunities, challenges and limitations. Nat Rev Cancer. 2009;9(8):550-562.

34. Cancer Genome Atlas Network. Comprehensive molecular portraits of human breast tumours. Nature. 2012;490(7418):61-70.

35. Hanker AB, Pfefferle AD, Balko JM, et al. Mutant PIK3CA accelerates HER2-driven transgenic mammary tumors and induces resistance to combinations of anti-HER2 therapies. Proc Natl Acad Sci U S A. 2013;110(35):14372-14377.

36. Gianni L, Bianchini G, Kiermaier A, et al. Neoadjuvant Pertuzumab (P) and Trastuzumab (H): Biomarker Analyses of a 4-Arm Randomized Phase II Study (NeoSphere) in Patients (pts) with HER2-Positive Breast Cancer (BC). Cancer Res. 2011;71(Suppl 24):S5-1.

37. Baselga J, Bradbury I, Eidtmann H, et al; NeoALTTO Study Team. Lapatinib with trastuzumab for HER2-positive early breast cancer (NeoALTTO): a randomised, open-label, multicentre, phase 3 trial. Lancet. 2012;379(9816):633-640.

38. Baselga J, Majewski I, Nuciforo PG, et al. PI3KCA mutations and correlation with pCR in the NeoALTTO trial (BIG 01-06). European Cancer Congress 2013 Abstract Book in the European Journal of Cancer. 2013:Abstract 1859.

39. Gwin WR1, Spector NL. Pertuzumab protects the achilles' heel of trastuzumab - emtansine. Clin Cancer Res. 2014;20(2):278-280.

40. Phillips GD, Fields CT, Li G, et al. Dual targeting of HER2-positive cancer with trastuzumab emtansine and pertuzumab: critical role for neuregulin blockade in antitumor response to combination therapy. Clin Cancer Res. 2014;20(2):456-468.

41. LoRusso PM, Weiss D, Guardino E, Girish S, Sliwkowski MX. Trastuzumab emtansine: a unique antibody-drug conjugate in development for human epidermal growth factor receptor 2-positive cancer. Clin Cancer Res. 2011;17(20):6437-6447.
Pharmacogenomics and Personalized Medicine

\section{Publish your work in this journal}

Pharmacogenomics and Personalized Medicine is an international, peerreviewed, open access journal characterizing the influence of genotype on pharmacology leading to the development of personalized treatment programs and individualized drug selection for improved safety, efficacy and sustainability. This journal is indexed on the American Chemical

\section{Dovepress}

Society's Chemical Abstracts Service (CAS). The manuscript management system is completely online and includes a very quick and fair peer-review system, which is all easy to use. Visit http://www.dovepress. com/testimonials.php to read real quotes from published authors. 